\title{
Health Care Analysis using Block Chain Technology
}

\author{
S. P. Balakannan, M. Maragatharajan, M. Venkatesulu
}

\begin{abstract}
Blockchain technology is an emerging area which connects all the functionalities of systems and computing. It converts all the information into chain of blocks and gives access rights to authenticated persons. So industries like healthcare, education and etc using this technology for confidential data processing. Especially in healthcare the patient record and drug management needs to be secure much. Here, we have proposed a method using blockchain to secure the information.
\end{abstract}

Key words: Blockchain, Healthcare, encryption.

\section{INTRODUCTION}

Blockchain is a technology that permits transactions to be gathered into blocked and recorded and cryptographically chains the blocks in chronological order [1]. It allows the resulting ledger to be accessed by different servers. Block chain satisfies three " T's" namely transparency, trust and transactions. Block chain allows bitcoin which is generated and circulated on the bitcoin network. Blockchain is not bitcoin, but it is the technology behind bitcoin. Bitcoin is the digital token and the blockchain is the ledger to keep track of who owns the digital tokens. We cannot have Bitcoin without blockchain, but we can have blockchain without Bitcoin. Bitcoin has accepted across the world bus has some limitations. The key features of blockchain include decentralized, distributed and transparent architecture of trust, automation and high security, immutability and traceability.

Most of the traditional business uses centralized ledger architecture. It leads to inefficient, expensive and vulnerable. But blockchain uses distributed ledger especially in the shared and synchronized areas like, transport, banking, financial auditing and retailing. The distributed ledger is a one that, all nodes have some level of access to that ledger [2]. All Nodes agree to a protocol that determines the "true state" of the ledger at any point in time. The application of this protocol is sometimes called "achieving consensus". The figure 1 illustrates the distributed ledger.

Revised Manuscript Received on December 16, 2019

S. P. Balakannan, Associate professor, Department of Information Technology, Kalasalingam Academy of Research and Education, Krishnankoil, India.

M. Maragatharajan, Assistant professor, Department of Information Technology, Kalasalingam Academy of Research and Education, Krishnankoil, India

M. Venkatesulu, Senior professor, Department of Information Technology, Kalasalingam Academy of Research and Education, Krishnankoil, India

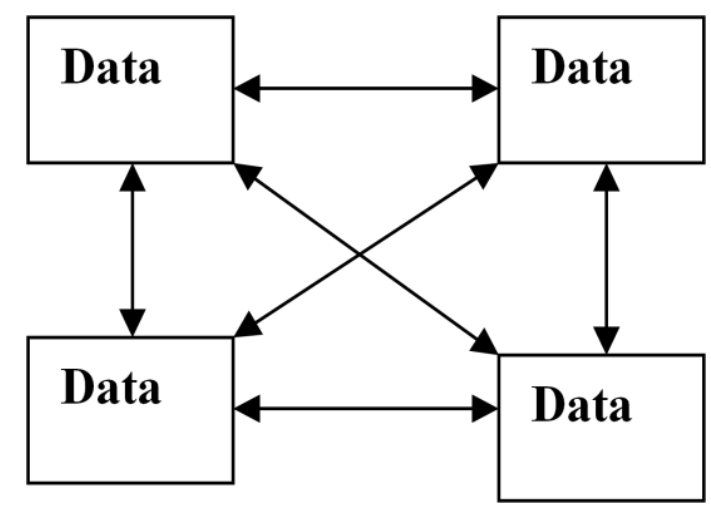

Figure 1 Distributed ledger

The working mechanism of distributed ledger will start from the initiation of user transactions. While the user initiate the transactions it will uses the digital signatures and user broadcast their transactions to nodes. Initiation and broadcasting of this transaction may use digital signature along with private or public keys. These transactions may be validated by more number of nodes. Then the nodes should aggregative validated and the transactions are converted as a chain of blocks. Now nodes broadcast blocks to each other. Now these blocks are get converted as chain of blocks using hash function.

There are two different kinds of proposals of distributed ledger namely open and closed or permission-less and permissioned. The methodology depends on degree of trust between nodes. Where there is no basis for trust, may be achieved through proof of work, which requires the algorithmic solving of a cryptographic hash. So blockchain is a type of distributed ledger in which value exchange transactions are sequentially grouped into blocks. Each block is chained to the previous block and immutably recorded across a peer-to-peer network, using cryptographic trust and assurance mechanisms.

Blockchain technology brings updated data, integrated information with time-stamped, Data auditability, immutability and transparency in transactions. The key features of blockchain in business are shared ledger, smart contract, privacy and consensus [3].

In blockchain, each block has two main components namely block header and list of transactions. The header consists of block reference number, block creation time and hash of previous block. Figure 2 illustrates the structure of block. 


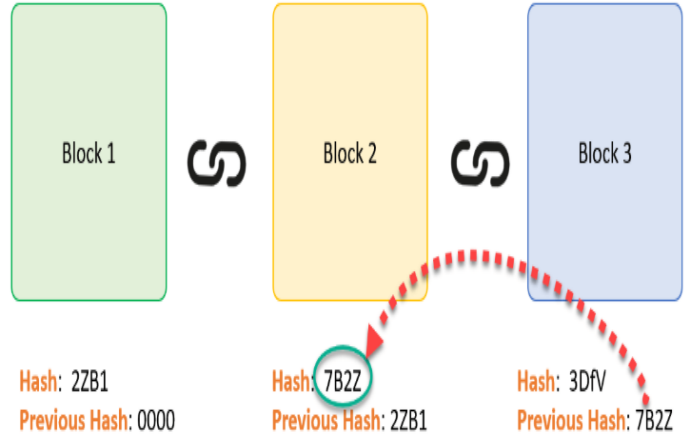

Figure 2 Structure of block

The hash function is a cryptographic algorithm, which change the original information into cipher text. It takes the input string of arbitrary length and produces fixed length output. It is a one way function used to detect changes in input. A person may request for a transaction. The transaction could involve crypto-currency, contracts, records or other information. The requested transaction is broadcasted to a P2P network with the help of nodes. The network of nodes validates the transaction and the user's status with the help of known algorithms. Once the transaction is complete the new block is then added to the existing Blockchain in such a way that is permanent and immutable

Blockchain offers proof-of-work. It is a computational problem that takes some efforts to solve. But time required to verify the results of the computational problem is very less when compared to the effort it takes to solve the computational problem itself [4].

The blockchain has applications in various domains. In healthcare industry it has remarkable benefits. The subsequent sections explain how healthcare management can be done using blockchain technology.

\section{HEALTHCARE MANAGEMENT USING BLOCKCHAIN TECHNOLOGY}

Healthcare management is an important function for efficient health care services. The basic concepts with respect to healthcare management are pharmacy arrangement, rational drug use, data collection, management and utilization of drugs and monitoring \& feedback services [5]. This model needs to be analyzed with relevant situation. Therefore, we can develop a system that connects all the modules of the system. Figure 3 shows use cases of healthcare industry.
The patient generated data consists of health apps, wearable devices, peripherals, active monitoring, health solutions and medical devices. Likewise the clinical data and health records contains handheld medical technology, various connected equipments, smart devices and other clinical devices. The combined activities of these produce blocks of patient data including their drug activities and etc.

The combined data will be encrypted using digital signature and hash functions. Theses encrypted information will be converted as number of blocks. Blockchain uses a distributed peer-peer network, and everyone is allowed to join. When someone enters this network, it will get the full copy of the Blockchain. When any user creates a new block, the new block is sent to all the users on the network. Each node needs to verify the block to make sure that it hasn't been altered. After verification, each node adds this new block to their Blockchain. All these nodes in this network create a consensus. They agree about what blocks are valid and which are not. Nodes in the network will reject blocks that are tampered with. So, to successfully tamper with a Blockchain

- One need to tamper with all blocks on the chain

- Redo the proof-of-work for each block

- Take control of greater than $50 \%$ of the peer-topeer network.

After doing all these, the tampered block becomes accepted by everyone else. But it is next to impossibility. The data blocks which are encrypted will be converted as a chain of blocks. The copies of these chains are available in data lakes.

Now, the data lakes stores different types of health data like images, genomics and lab records. It consists of structured and unstructured data. So the information is encrypted and digitally signed. Likewise, blockchain of this healthcare network consists of a complete indexed history, patient's unique identifier and an encrypted link to the health record. Each record is time stamped. All patient records including their history are chained together. Patients have control over their health records.

The authorized persons can decrypt the data using digital signature. This data can be used for health analytics and Internet of Medical things (IoMT). It is a huge revolution of healthcare. It is a collection of medical devices and its applications which connect to healthcare IT systems. The IoMT provide the data to either patients or providers like doctors.

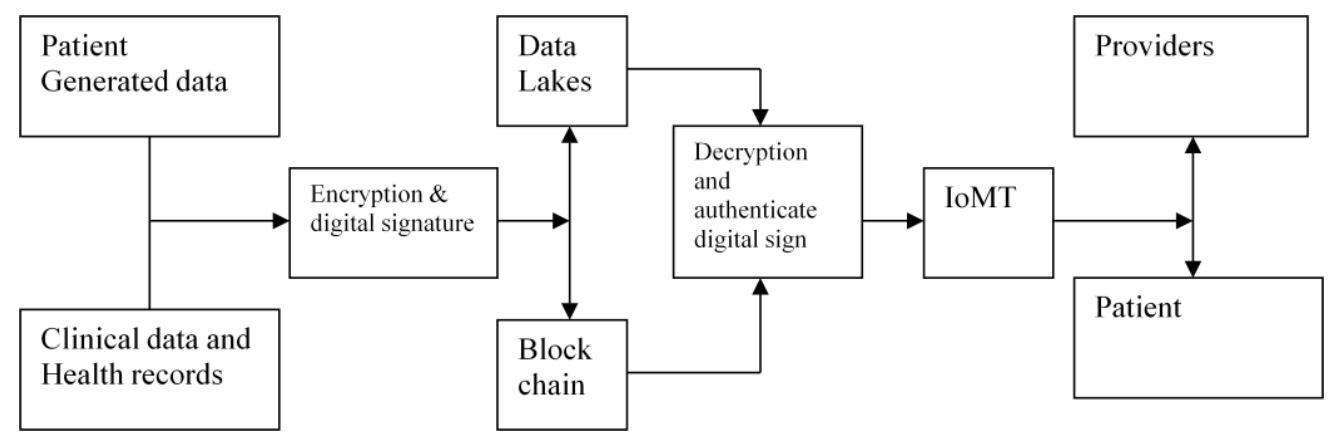

Figure 3 Blockchain Technology - Use cases for healthcare industry 
The patients use mobile devices to grant access permission to data by providing public key. Similarly the providers use health applications to access health data. The blockchain network consensus enables disintermediation to automate claim adjudication and payment processing with predefined smart contracts.

\section{HEALTHCARE SYSTEMS WITH AND WITHOUT BLOCKCHAIN TECHNOLOGY}

Healthcare systems may refuse to relay valid transactions of a node. Sometimes, an attacker may attempt to create blocks that include or exclude specific transactions. But invention of blockchain relaxes these issues. Also digitization of health economy and advances in data science and machine learning has led to numerous applications in healthcare industry .Blockchain Technology enhances the trust and gives transparency in value chains. It reduces the trade cost. Blockchain allows us to make the chains harder by adding more cryptographic functions.

\section{CONCLUSION}

Blockchain is a new technology which uses distributed ledger technology and allows digital records and information to be shared in secure manner with transparency. The major steps involved in healthcare data transmissions are requesting of data, the data to be broadcasted to the peer-to-peer network, validation of data and addition to the blockchain. All these steps are done in an unalterable way. Here encryption, Hash algorithms and keys play vital role.

The blockchain is not suited to all situations. It will work better where multiple entities are involved in a transaction. Also in many circumstances, it may create problems if it is used for transactions that do not require high level of reliability.

But in case of heath care management, it works better because of involvement of patients, doctors, drugs advisors, medical devices and etc. Blockchain technology promises high hope in healthcare management at all levels such as patients, doctors, clinical hospitals and Pharmaceutical companies etc.,.

\section{REFERENCES}

1. Emmanuelle Ganne, "Blockchain revolutionize international trade" world trade organization, 2018.

2. Antony Lewis, "Understanding blockchain Technology" DBS Asian sights, 2016.

3. Michael Crosby, "Blockchain Technology-Beyond bitcoin" University of California, 2015.

4. Antony Lewis, "A gentle introduction to blockchain" Brave new coin, 2017

5. Nancy Lio, "A brief introduction to Blockchain Technology" Yale center for computer science, 2015.

\section{AUTHORS PROFILE}

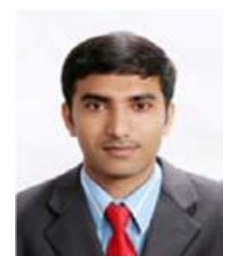

Balakannan S.P received his Ph.D. degree from the Department of Electronics and Information Engineering at Chonbuk National University, South Korea (2010). He has received his master degree (5 years integrated) from the Department of Computer Science and Engineering, Bharathiar University, India, in the year 2003. He has worked as a Project Assistant in Indian Institute of Technology (IIT), Kharagpur, India from 2003 to 2006. Currently, he is working as Assistant Professor in the Department of Information Technology, Kalasalingam University, Tamilnadu, India. His areas of interest include Wireless Network, Network Coding, Cloud \& Green Computing, Cryptography, and Mobile Communication.

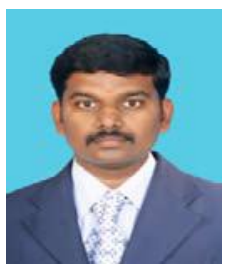

Maragatharajan $\mathbf{M}$ received his Bachelor degree in Electronics \& Communication Engineering from Anna University by 2007. He has received his Master degree in Information Technology from Kalasalingam University, 2010 and completed his $\mathrm{Ph} . \mathrm{D}$ in the area of MANET. He has worked as a Project Associate in TIFAC CORE in Network Engineering, Kalasalingam University from 2007 to 2008. Currently, He is working as an Assistant Professor in the Department of Information Technology, Kalasalingam University. His areas of interest are Ad-hoc Networks, Wireless Networks, and Network Security.

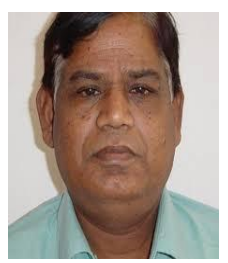

Dr.M.Venkatesulu did his under graduation in Mathematics from the University of Madras, Post graduation in Mathematics in Mathematics from Sri Venkateswara University, Tirupati and obtained PhD in Mathematics from the Indian Institute of Technology, Kanpur. He was a faculty member in the department of mathematics and Computer Science at Sri Sathya Sai Institute of Higher Learning ( Deemed to be University), Prasanthinilayam, Andrapradesh, India.

He worked as a consultant for a short duration in Satyam Computer Services Limited, Secunderabad, India during 2002. He has joined Kalasalingam Academy of Research and Education in 2004 as Senior Professor in Mathematics. He was a visiting Professor in the Department of Mathematics and Statistics, University of Missouri, Kansas City, USA during 2006-2007. He has published more than 70 papers in peer reviewed journals and Conferences and guided 14 PHDs in Mathematics and Computer Science areas. He has completed $4 \mathrm{R} \& \mathrm{D}$ projects funded by RDDO and DST, Government of India. He is a reviewer for 2 international journals published by Elsevier. 